\title{
Validation of Addenbrooke's cognitive examination III for detecting mild cognitive impairment and dementia in Japan
}

Shintaro Takenoshita, Seishi Terada*, Hidenori Yoshida, Megumi Yamaguchi, Mayumi Yabe, Nao Imai, Makiko Horiuchi, Tomoko Miki, Osamu Yokota and Norihito Yamada

\begin{abstract}
Background: Early detection of mild cognitive impairment $(\mathrm{MCl})$ and dementia is very important to begin appropriate treatment promptly and to prevent disease exacerbation. We investigated the screening accuracy of the Japanese version of Addenbrooke's Cognitive Examination III (ACE-III) to diagnose MCl and dementia.

Methods: The original ACE-III was translated and adapted to Japanese. It was then administered to a Japanese population. The Hasegawa Dementia Scale-revised (HDS-R) and Mini-mental State Examination (MMSE) were also applied to evaluate cognitive dysfunction. In total, 389 subjects (dementia $=178, \mathrm{MCl}=137$, controls $=73$ ) took part in our study.

Results: The optimal ACE-III cut-off scores to detect MCl and dementia were 88/89 (sensitivity 0.77, specificity 0.92) and 75/76 (sensitivity 0.82 , specificity 0.90 ), respectively. ACE-III was superior to HDS-R and MMSE in the detection of $\mathrm{MCl}$ or dementia. The internal consistency, test-retest reliability, and inter-rater reliability of ACE-III were excellent.
\end{abstract}

Conclusions: ACE-III is a useful cognitive test to detect $\mathrm{MCl}$ and dementia. ACE-III may be widely useful in clinical practice.

Keywords: Addenbrooke's cognitive examination, Cognitive screening, Diagnosis dementia, Diagnosis mild cognitive impairment, Mild cognitive impairment

\section{Background}

Early detection of cognitive deterioration in the prodromal stage of dementing diseases is arguably important in order to initiate curable treatments in the future. Mild cognitive impairment (MCI) converts to dementia at a rate of $\sim 10 \%$ per year [1], but its clinical diagnosis is a challenge due to the variety and often dynamic nature of symptoms [2]. Nonetheless, a reliable and valid test to detect MCI in clinical settings has not been developed [3].

$\mathrm{ACE}$ and its revised version (ACE-R) were created as concise tests for detecting mild cognitive dysfunction $[4$, 5]. ACE-R has been translated for use in a number of non-English-speaking countries worldwide and widely

\footnotetext{
* Correspondence: terada@okayama-u.ac.jp

Department of Neuropsychiatry, Okayama University Graduate School of Medicine, Dentistry and Pharmaceutical Sciences, 2-5-1 Shikata-cho, Kita-ku, Okayama 700-8558, Japan
}

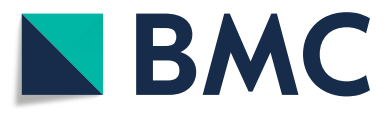

(c) The Author(s). 2019 Open Access This article is distributed under the terms of the Creative Commons Attribution 4.0 International License (http://creativecommons.org/licenses/by/4.0/), which permits unrestricted use, distribution, and reproduction in any medium, provided you give appropriate credit to the original author(s) and the source, provide a link to the Creative Commons license, and indicate if changes were made. The Creative Commons Public Domain Dedication waiver (http://creativecommons.org/publicdomain/zero/1.0/) applies to the data made available in this article, unless otherwise stated.

adopted in clinical and research settings [6]. A study at our facility verified the Japanese version [7]. Despite its widespread use, ACE- $\mathrm{R}$ is relatively weak in several domains, such as repetition, comprehension, and visuospatial items $[2,6]$. Healthy older adults often fail the repetition item on ACE-R due to poor hearing or a short attention span [8]. Comprehension items on ACE-R exhibit poor sensitivity to cognitive impairment because individuals with cognitive dysfunction often show scores in the normal range [9]. In addition, some changes in ACE- $\mathrm{R}$ fail to accurately reflect the original ACE. For example, spelling of the word "WORLD" backwards can be substituted for subtraction of serial $7 \mathrm{~s}$ from 100 in ACE-R, but these two items are known to present different challenges [10]. Most importantly, ACE-R included several elements of the MMSE. Due to copyright issues, it has become difficult to keep using ACE-R. [11] 
Therefore, the original authors developed a new version of ACE, namely ACE-III [6]. ACE-III is also scored on a total of 100 and contains five cognitive domains. To date, versions of ACE-III in many different languages have been validated [12-15], and we recently created a Japanese version of ACE-III.

In this study, we hypothesized that ACE-III would be superior to the conventional Hasegawa Dementia Scale-revised (HDS-R) and Mini-mental State Examination (MMSE) in detecting MCI and dementia in a Japanese population. Our objective therefore was to (1) provide detailed normative data for the sub- and total scores on ACE-III; (2) decide the optimal cut-off scores of ACE-III to identify MCI or dementia, and compare its validity with that of MMSE or HDS-R; and (3) evaluate the test-retest and inter-rater reliabilities and internal consistency.

\section{Methods}

\section{Participants}

A total of 389 subjects at the Memory Clinic of Okayama University Hospital between January 2013 and March 2017 who fulfilled the following criteria were included in this study (Table 1). All subjects (i) received general physical and neurological examinations and laboratory testing, including syphilis serology, plasma vitamin B1, serum vitamin B12, and thyroid function tests; (ii) took MMSE [16, 17]' and HDS-R [18, 19]; and (iii) received magnetic resonance imaging (MRI) and/or computed tomography (CT) of the head. The exclusion criteria were (i) the presence of delirium or (ii) the existence of psychiatric diseases.

The profile of each subject (sex, age, years of education) was checked. Neuropsychological examinations were performed by clinical psychologists specialized in dementia, and the Clinical Dementia Rating (CDR) [20] score was determined by the chief clinician. When all examinations had been completed, two or more geriatric psychiatrists and two or more experienced clinical psychologists conferred, and the clinical diagnosis was established independent of the performance on ACE-III.

A total of 389 subjects were divided into three groups: a dementia group $(n=178)$, an MCI group $(n=137)$, and a control group $(n=74)$.

All patients diagnosed with dementia had a dementia severity of 0.5 (suspicious) or 1 (mild) based on the CDR. Patients in the dementia group were diagnosed with Alzheimer's disease dementia (ADD; $n=$ 131), dementia with Lewy bodies (DLB; $n=21$ ), behavioral variant frontotemporal dementia (bvFTD; $n=$ 9), vascular dementia $(\mathrm{VaD} ; n=4)$, and others $(n=$ 13). Patients with ADD were diagnosed with probable $\mathrm{AD}$ according to the criteria formulated by the $\mathrm{Na}$ tional Institute on Aging-Alzheimer's Association [21]. Patients with DLB, FTD, or VaD were diagnosed in accordance with the DLB diagnostic criteria formulated by McKeith et al. [22], the FTDC criteria for bvFTD [23], and the American Heart Association/ American Stroke Association guidelines for $\mathrm{VaD}$ [24], respectively.

Patients of the MCI group fulfilled the criteria of (1) concern about a deficit in cognition compared with the person's previous level; (2) performance that is lower than would be expected for the patient's age and educational background $(\mathrm{CDR}$ score $=0.5)$ in one or more cognitive domains; (3) no or minimal disturbance in activities of daily living, as established by an interview with the patient and an informant [25]; and (4) being not

Table 1 Comparison of demographic data, MMSE, HDS-R, ACE-III total and subscores in control, MCl, and dementia groups $(n=389$, standard deviation in parenthesis)

\begin{tabular}{|c|c|c|c|c|c|c|}
\hline & $\begin{array}{l}\text { CONTROL } \\
n=74\end{array}$ & $\begin{array}{l}\mathrm{MCl} \\
n=137\end{array}$ & $\begin{array}{l}\text { DEMENTIA } \\
n=178\end{array}$ & $\begin{array}{l}D \text { vs. } C \\
P \text { values }\end{array}$ & $\begin{array}{l}D \text { vs. M } \\
P \text { values }\end{array}$ & $\begin{array}{l}M \text { vs. } C \\
P \text { values }\end{array}$ \\
\hline Sex, men/women & $30 / 44$ & $71 / 66$ & $66 / 112$ & n.s. & * & n.s. \\
\hline Age & $72.1(7.1)$ & $75.3(8.3)$ & $78.6(7.2)$ & $* * *$ & $* * *$ & * \\
\hline Education, years & $12.9(2.3)$ & $13.0(2.7)$ & $12.0(2.4)$ & $*$ & $* *$ & n.s. \\
\hline MMSE, 0-30 & $28.5(1.6)$ & $25.5(2.4)$ & $21.7(3.3)$ & *** & $* * *$ & *** \\
\hline HDS-R, 0-30 & $27.8(2.2)$ & $23.5(3.3)$ & $18.7(3.8)$ & $* * *$ & $* * *$ & *** \\
\hline ACE-III total score, 0-100 & $93.5(3.4)$ & $82.7(7.2)$ & $66.0(11.4)$ & $* * *$ & $* * *$ & $* * *$ \\
\hline Attention and Orientation, 0-18 & $17.3(1.1)$ & $16.0(1.8)$ & $13.0(2.7)$ & $* * *$ & $* * *$ & *** \\
\hline Memory, 0-26 & $23.6(2.2)$ & $16.8(4.2)$ & $9.8(4.3)$ & $* * *$ & $* * *$ & *** \\
\hline Fluency, 0-14 & $11.4(1.7)$ & $10.2(2.3)$ & $7.8(2.7)$ & $* * *$ & $* * *$ & ** \\
\hline Language, $0-26$ & $25.6(0.8)$ & $24.6(1.7)$ & $21.8(3.9)$ & $* * *$ & $* * *$ & n.s. \\
\hline Visuospatial, 0-16 & $15.6(0.7)$ & $15.1(1.4)$ & $13.6(2.9)$ & $* * *$ & $* * *$ & n.s. \\
\hline
\end{tabular}

MMSE Mini-mental State Examination, HDS-R Hasegawa Dementia Scale-revised, ACE-III Addenbrooke's Cognitive Examination-III, MCI mild cognitive impairment, $D$ dementia, $C$ control, $M \mathrm{MCl}$, n.s. not significant

${ }^{*} P<0.05,{ }^{* *} P<0.01,{ }^{* * *} P<0.001$. Pairwise comparisons were performed using Bonferroni's test 
sufficiently functionally and cognitively impaired to meet the DSM-IV-TR criteria for dementia [26].

Seventy-four subjects with no decline in cognition compared with their previous level $(\mathrm{CDR}$ score $=0)$ were used as a control group. None had evidence of organic dementing disorders or psychiatric diseases, and all had no impairment in their activities of daily living (ADL) and instrumental ADL.

\section{Instruments}

Like ACE-R, ACE-III is composed of five domains and each represents a certain cognitive function: (1) orientation and attention (18 points), (2) memory (26 points), (3) fluency (14 points), (4) language (26 points), and (5) visuospatial function (16 points). The highest possible score on ACE-III is 100 points, and lower scores indicate worse cognitive functioning.

The differences between ACE-R and ACE-III are as follows $[2,6]$. In the attention domain of ACE-R, serial subtraction of $7 \mathrm{~s}$ from 100 could be replaced by spelling the word 'WORLD' backwards. However, in ACE-III, the option of spelling 'WORLD' backwards was removed, and only subtraction of serial $7 \mathrm{~s}$ from 100 is performed $[2,6]$. In the language domain, the three-step command was changed to the three single-step commands that increase in syntactical complexity. ${ }^{2}$ Comprehension of the written command ('close your eyes') was taken out. The sentence-writing task was modified, and participants are asked to write two or more sentences on a single topic for a maximum score of 2 points [2]. The phrase repetition items were replaced by the repetition of two common proverbs [2]. Overlapping infinity loops replaced the intersecting pentagons in the visuospatial section [2]. The memory and fluency domains in ACE-R were not modified. The translation and modification of ACE-R into the Japanese version was previously reported in detail [7]. The Japanese version of ACE-III (ACE-III-J) was modified to reflect the English version of ACE-III.

MMSE is a concise cognition screening test. It includes a series of items that measure orientation, recall, language, and visual construction [16, 17]. The full score of MMSE is 30 points. HDS-R assesses cognitive function of orientation, memory, attention/calculation, delayed recall, and verbal fluency $[18,19]$. This is a reliable and brief instrument to evaluate global cognitive function. The maximum total possible score is 30 points.

\section{Reliability}

Inter-rater reliability was measured by determining the intraclass correlation coefficient (ICC) of 25 consecutive patients. Two clinical psychologists assessed subjects at the same time, and they were blind to each other's scores. One of them actively assessed ten patients while the other passively observed, and their roles were reversed for the other 15 . We evaluated test-retest reliability using the ICC of 26 consecutive patients. The second session for test-retest reliability was done four to eight weeks after the first session. We evaluated the internal consistency reliability within ACE-III-J using Cronbach's coefficient alpha [27].

\section{Statistical analysis}

Statistical analyses were performed using the IBM SPSS Statistics 23.0 software program. A value of $P<0.05$ was accepted as significant. Two groups were compared by independent sample t-tests. Three groups were compared using one-way analysis of variance, followed by Bonferroni correction at the time of post hoc analysis. $X^{2}$ tests were used for comparison of categorical data (gender). We used a multiple regression analysis to examine possible associations of the clinical characteristics (gender, age, and years of education) with the total ACE-III score.

We determined the sensitivity and specificity of ACE-III, MMSE, and HDS-R using a receiver operating characteristic (ROC) curve [7]. We used the area under the curve (AUC) as a scale of each test's ability to differentiate between groups of participants (dementia vs. MCI and normal; MCI vs. normal).

In this study, we used StAR software to assess statistical differences between AUCs of the three tests [28]. The most suitable cut-off scores for identifying dementia and MCI were determined as the scores that led to the maximal accuracy of classification. Subsequently, positive predictive values (PPV) and negative predictive values (NPV) were estimated at different prevalence rates $(5,10,20$, and $40 \%)$ for each optimal cutoff score.

Correlation between the CDR sum of box (CDR SoB) score and ACE-III scores was evaluated using Spearman's correlation coefficient. A value of $P<0.05$ was accepted as significant.

\section{Results \\ Clinical characteristics of dementia, $\mathrm{MCl}$, and control groups}

Table 1 shows the clinical characteristics, MMSE scores, HDS-R scores, and ACE-III-J total and subdomain scores of dementia, $\mathrm{MCI}$, and control groups.

Age $(F(2,386)=20.93, P<0.001)$ and years of education (F $(2,386)=7.47, P=0.001)$ were significantly different between the three groups. The dementia group was significantly older and less educated than the control and MCI groups, and the MCI group was older than the control group. The multiple regression analysis showed that age $(\beta$; standard partial regression coefficient $=-0.282, P<0.001)$ and education $(\beta=0.129, P<$ $0.05)$ had a significant impact on the ACE-III-J score. When the same analysis was done on the normal 
controls $(n=74)$, it revealed that only age $(\beta=-0.266, P$ $<0.05$ ) affected ACE-III-J performance significantly.

ACE-III-J total $(\mathrm{F}(2,386)=288.562, P<0.001)$, MMSE $(\mathrm{F}(2,386)=184.793, P<0.001)$, and HDS-R $(\mathrm{F}(2,386)$ $=189.996, P<0.001)$ scores were significantly different between the three groups. On ACE-III-J, scores of all five subdomains differed significantly among the three groups. According to the post hoc analysis with Bonferroni correction, the control and MCI groups had higher scores in all five domains than the dementia group $(P<$ 0.001). The control group had higher scores than the MCI group in attention/orientation, memory, and fluency domains, but the differences between the two groups in language and visuospatial scores were not significant.

\section{Demographics of dementia group (very mild and mild)}

The dementia group $(n=178)$ was subdivided into two groups, very mild $(\mathrm{CDR}=0.5)$ and mild $(\mathrm{CDR}=1)$, according to the CDR score. The clinical characteristics are shown in Table 2.

There were no significant differences in education or gender distribution between the groups. The mild dementia group was significantly older than the very mild dementia group $(P<0.05)$ and had significantly lower scores than the very mild dementia group on ACE-III-J, MMSE, and HDS-R $(P<0.001)$. On four of the subscores of the ACE-III-J, excluding the memory score, the mild dementia group had significantly lower scores than the very mild dementia group.

Table 2 Comparison of demographic data, MMSE, HDS-R, ACEIII total and subscores in very mild (CDR 0.5) and mild (CDR 1) dementia groups ( $n=178$, standard deviation in parenthesis)

\begin{tabular}{llll}
\hline & VERY MILD & MILD & $P$ \\
& $n=113$ & $n=65$ & values \\
\hline Sex, men/women & $43 / 70$ & $23 / 42$ & n.S. \\
Age & $77.6(7.7)$ & $80.4(6.0)$ & $*$ \\
Education, years & $11.8(2.3)$ & $12.3(2.5)$ & n.s. \\
MMSE, 0-30 & $22.5(3.0)$ & $20.2(3.1)$ & $* * *$ \\
HDS-R, 0-30 & $19.7(3.3)$ & $16.9(4.0)$ & $* * *$ \\
ACE-III total score, 0-100 & $68.7(10.2)$ & $61.1(11.8)$ & $* * *$ \\
$\quad$ Attention and Orientation, 0-18 & $13.8(2.4)$ & $11.7(2.6)$ & $* * *$ \\
Memory, 0-26 & $10.1(4.2)$ & $9.1(4.3)$ & n.s. \\
Fluency, 0-14 & $8.2(2.5)$ & $7.1(3.0)$ & $*$ \\
Language, 0-26 & $22.5(3.4)$ & $20.5(4.4)$ & $* *$ \\
Visuospatial, 0-16 & $14.1(2.4)$ & $12.7(3.3)$ & $* *$ \\
\hline
\end{tabular}

MMSE Mini-mental State Examination, HDS-R, Hasegawa Dementia Scalerevised, $A C E$-III Addenbrooke's, Cognitive Examination-III; CDR clinical dementia rating, n.s. not significant

${ }^{*} P<0.05,{ }^{* *} P<0.01,{ }^{* * *} P<0.001$

\section{Normative data}

Normative scores were generated for the ACE-III-J total and subdomain scores using data of the control group, based on the mean minus two standard deviations (lower limits of normal) for three age bands $(\leq 69,70$ 79 , and $\geq 80$ years old) as well as all age groups, as shown in Table 3.

Among the three age groups, the number of years of education differed $(F(2,71)=5.228, P<0.01)$. By post hoc analysis, the $\leq 69$ age group had more years of education than the 70-79 and $\geq 80$ age groups (respectively, $P=0.036$ and 0.016 ).

Among these three age groups, ACE-III-J total scores (F $(2,71)=3.857, P<0.05)$ and visuospatial subscores $(\mathrm{F}$ $(2,71)=4.031, P<0.05)$ differed. No significant differences were found between the three groups in the attention/orientation $(\mathrm{F}(2,71)=0.279, P=0.757)$, memory $(\mathrm{F}$ $(2,71)=1.173, P=0.315)$, fluency $(\mathrm{F}(2,71)=1.900 P=$ $0.157)$, and language $(F(2,71)=0.174, P=0.841)$ subscores. In the total ACE-III-J score, post hoc analysis disclosed no significant difference among the three groups. The scores of the $\leq 69$ age group were significantly higher than those of the $\geq 80$ age group $(P<0.05)$ in the visuospatial domain.

\section{Diagnostic interpretation}

The ROC curves of ACE-III-J, HDS-R, and MMSE for diagnosing $\mathrm{MCI}$ or dementia are shown in Fig. 1 (for MCI in Fig. 1a, and for dementia in Fig. 1b). The AUCs of ACE-III-J, HDS-R, and MMSE for diagnosing MCI were 0.914 (0.876-0.953), 0.859 (0.807-0.912), and 0.838 (0.780-0.896), respectively. The AUC of ACE-III-J was significantly larger than those of HDS-R and MMSE (ACE-III-J vs. HDS-R, $P<0.05$; ACE-III-J vs. MMSE, $P$ $<0.01)$. The most suitable cut-off score of ACE-III-J for discriminating MCI patients from controls was 88/89 (sensitivity 0.77, specificity 0.92), and those of the HDS-R and MMSE were 24/25 (sensitivity 0.57, specificity 0.89 ) and $26 / 27$ (sensitivity 0.61 , specificity 0.86 ), respectively. The PPV and NPV of ACE-III for identifying $\mathrm{MCI}$ at different prevalence rates were 5\% (PPV 0.35, NPV 0.99), 10\% (PPV 0.52, NPV 0.97), 20\% (PPV 0.72, NPV 0.94), and 40\% (PPV 0.87, NPV 0.86) (Table 4).

The AUCs of ACE-III-J, HDS-R, and MMSE for diagnosing dementia were $0.938 \quad(0.915-0.960), 0.881$ (0.847-0.915), and $0.881(0.847-0.914)$, respectively. The AUC of ACE-III-J was significantly larger than those of HDS-R and MMSE (ACE-III-J vs. HDS-R, $P<0.001$; ACE-III-J vs. MMSE, $P<0.001)$. For discriminating dementia patients from MCI patients and controls, the most suitable cut-off score of ACE-III-J was 75/76 (sensitivity 0.82 and specificity 0.90 ), and those of HDS-R and MMSE were 20/21 (sensitivity 0.67 , specificity 0.89 ) and $23 / 24$ (sensitivity 0.64 , specificity 0.87 ), respectively. 
Table 3 Lower limit of normal (cut-off scores) of ACE-III total and subscores for age bands $(<69,70-79,>80$ years old) and all age groups (control mean - 2 standard deviations)

\begin{tabular}{|c|c|c|c|c|c|c|c|c|}
\hline AGE & $\mathrm{N}$ & EDUCATION & TOTAL & ATTENTION & MEMORY & FLUENCY & LANGUAGE & VISUO \\
\hline (range) & & (mean, years) & $(\max .100)$ & (max. 18) & (max. 26) & (max. 14) & (max. 26) & $(\max .16$ \\
\hline-69 & 26 & 14.0 & 89 & 16 & 20 & 10 & 25 & 16 \\
\hline 70-79 & 39 & 12.6 & 87 & 16 & 20 & 8 & 24 & 14 \\
\hline $80-$ & 9 & 11.6 & 85 & 15 & 19 & 8 & 24 & 14 \\
\hline All & 74 & 12.9 & 87 & 16 & 20 & 8 & 24 & 15 \\
\hline
\end{tabular}

ACE-III Addenbrooke's Cognitive Examination-III, Visuo, visuospatial

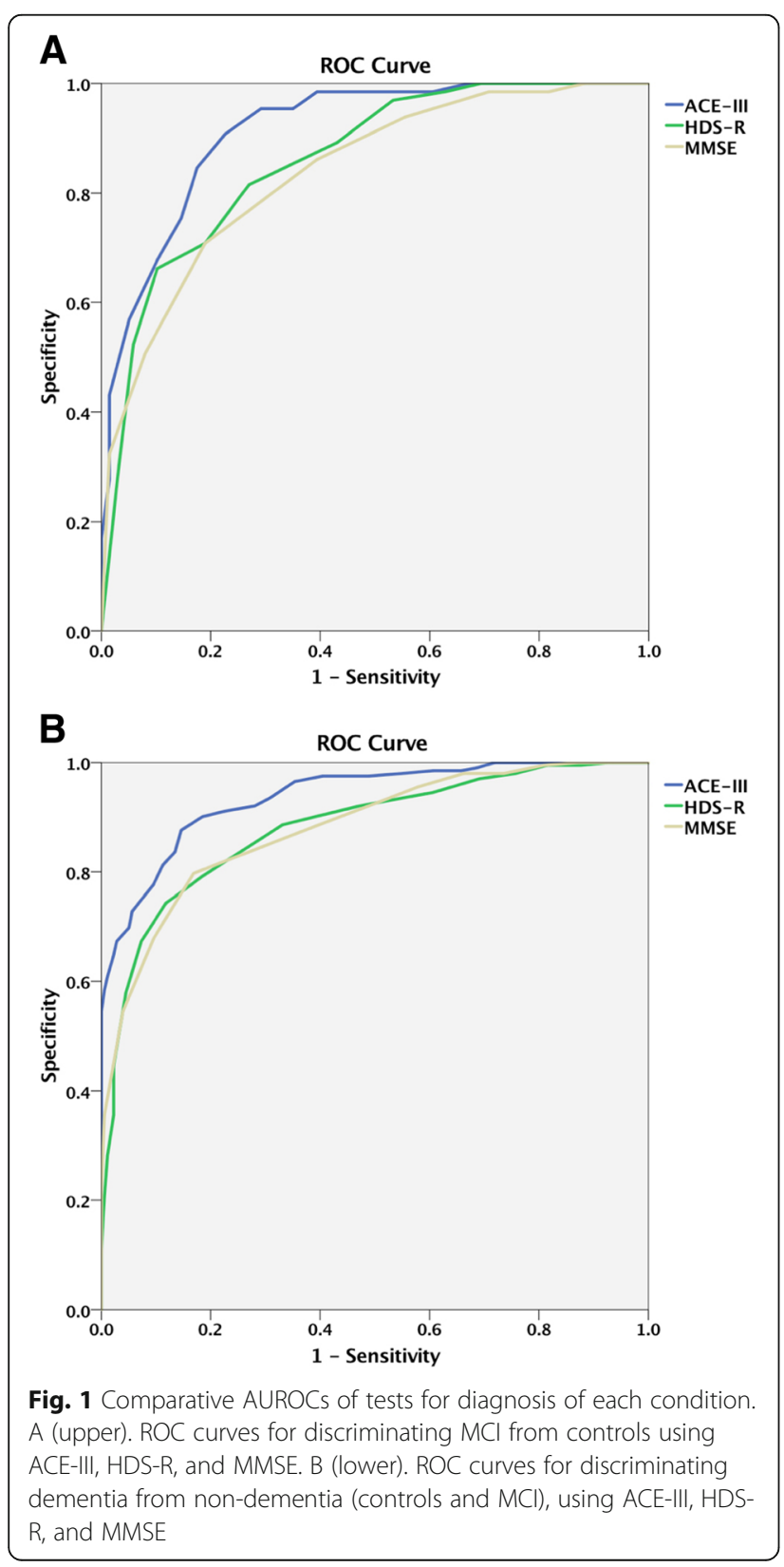

The PPV and NPV of ACE-III for identifying dementia at different prevalence rates were 5\% (PPV 0.30, NPV 0.99), 10\% (PPV 0.48, NPV 0.98), 20\% (PPV 0.67, NPV 0.95), and 40\% (PPV 0.85, NPV 0.88) (Table 4).

\section{Reliability}

The inter-rater reliability of ACE-III-J was very good, with an ICC of 0.996. The test-retest reliability of ACE-III-J was also very good ( $\mathrm{ICC}=0.918)$. The internal consistency of ACE-III-J was high (Cronbach's coefficient $\alpha=0.870$ ).

\section{ACE-III scores and CDR sum of boxes}

Spearman's correlation analysis of the scores of the CDR $\mathrm{SoB}$ and the ACE-III scores revealed that there was a significant correlation between them (correlation coefficient $=-0.396, p<0.001)$ in MCI patients.

\section{Discussion}

The reliability of ACE-III-J was excellent. ACE-III-J was found to be a sensitive and specific screening test to diagnose MCI and dementia in a Japanese sample, and it was better than the MMSE and HDS-R in accuracy for identifying MCI and dementia. These results suggest that ACE-III-J is a reliable and valid screening instrument.

Although ACE-III-J takes slightly longer to perform than MMSE and HDS-R, it evaluates a broader range of cognitive functions than MMSE and HDS-R, particularly in the domains of memory, language, and visuospatial components [7]. Thus, we consider that ACE-III-J provides a more useful and precise instrument than MMSE and HDS-R for diagnosing MCI and dementia. However, in 19 of the subjects, screening results for dementia are positive in MMSE but negative in ACE-III. Six of the 19 persons were diagnosed as dementia. Even if a person takes a score that exceeds the cut-off score in ACE-III, it is necessary to consider the possibility of dementia if the MMSE score of the person is below the cut-off score for dementia in MMSE.

Several non-English versions of the ACE-III have been reported [12, 14, 15, 29]. The mean scores of controls in various studies were 95.4 points (mean age 66.1 years, 
Table 4 Sensitivity, specificity and positive predictive values (PPV) at different prevalence rates of optimal cut off ACE-III, HDS-R, and MMSE scores for identifying $\mathrm{MCl}$ and dementia (Values in parentheses represent the respective negative predictive value)

\begin{tabular}{|c|c|c|c|c|c|c|c|}
\hline \multirow[t]{2}{*}{ TEST } & \multirow{2}{*}{$\begin{array}{l}\text { CUT-OFF } \\
\text { SCORE }\end{array}$} & \multicolumn{2}{|l|}{$\mathrm{MCl}$} & \multicolumn{4}{|c|}{ PPV at different prevalence rates } \\
\hline & & SENSITIVITY & SPECIFICITY & $5 \%$ & $10 \%$ & $20 \%$ & $40 \%$ \\
\hline ACE-III & $88 / 89$ & 0.77 & 0.92 & $0.35(0.99)$ & $0.52(0.97)$ & $0.72(0.94)$ & $0.87(0.86)$ \\
\hline HDS-R & $24 / 25$ & 0.57 & 0.89 & $0.22(0.98)$ & $0.37(0.95)$ & $0.57(0.89)$ & $0.78(0.76)$ \\
\hline MMSE & $26 / 27$ & 0.61 & 0.86 & $0.19(0.98)$ & $0.33(0.95)$ & $0.52(0.90)$ & $0.75(0.77)$ \\
\hline \multirow[t]{2}{*}{ TEST } & CUT-OFF & \multicolumn{2}{|c|}{ DEMENTIA } & \multicolumn{4}{|c|}{ PPV at different prevalence rates } \\
\hline & SCORE & SENSITIVITY & SPECIFICITY & $5 \%$ & $10 \%$ & $20 \%$ & $40 \%$ \\
\hline ACE-III & $75 / 76$ & 0.82 & 0.90 & $0.30(0.99)$ & $0.48(0.98)$ & $0.67(0.95)$ & $0.85(0.88)$ \\
\hline HDS-R & $20 / 21$ & 0.67 & 0.89 & $0.24(0.98)$ & $0.39(0.96)$ & $0.59(0.91)$ & $0.80(0.80)$ \\
\hline MMSE & $23 / 24$ & 0.64 & 0.87 & $0.21(0.98)$ & $0.36(0.96)$ & $0.55(0.91)$ & $0.77(0.78)$ \\
\hline
\end{tabular}

ACE-III Addenbrooke's Cognitive Examination-III, HDS-R Hasegawa Dementia Scale-revised, MMSE Mini-mental State Examination

education 13.9 years) on the English version [6], 96.7 points (mean age 66.6 years) on the English version [30], 89.4 points (mean age 70.4 years, education 6.2 years) on the Portuguese version [12], 89.4 points (mean age 65.8 years, education 12.4 years) on the Spanish version [29], 89.3 points (mean age 68.7 years, education 11.5 years) on the Chinese version [15], and 93.5 points (mean age 72.1 years, education 12.9 years) on the Japanese version. Age and years of education had significant effects on the total ACE-III score, as shown in several studies including ours $[15,29]$, and those two factors might explain the differences in mean scores to some extent. The total and subscores of the control group of ACE-III-J were almost identical to those of the original English version. The mean scores of ACE-III-J vs. the original ACE-III were 93.5 vs. 95.4 in total score, 17.4 vs.17.3 in the attention domain, 25.6 vs. 25.6 in the language domain, and 15.6 vs. 15.6 in the visuospatial domain. The memory and fluency subscores in the original English version were not reported in the first paper [6].

The cut-off scores of the original ACE-III scores were almost identical to those of the original ACE-R [6]. Thus, the Japanese version of ACE-III was equivalent to the original English version as a cognitive screening instrument. The optimal cut-off score for identifying MCI $(88 / 89)$ in this study was similar to the original higher cut-off score (88) for identifying dementia. The cut-off score for identifying dementia (75/76) in this study was lower than the original lower cut-off score (82). The original study for ACE-R compared dementia patients with normal controls rather than MCI patients [5]. In this study, we set an optimal cut-off score to differentiate dementia patients from MCI patients. The difference in comparative groups tested to create the cut-off scores of English and Japanese versions may have caused the difference in cut-off scores.

The sensitivities reported by other studies in which the sensitivity of the dementia diagnosis was evaluated are higher than that $(0.82)$ in this report. In particular, Elamin et al. reported that the sensitivity for the diagnosis of dementia was 0.915 , and Wang et al. reported that the sensitivity was 0.911 . However, they calculated the sensitivity in distinguishing dementia patients from cognitively normal subjects or patients with subjective memory impairment in previous reports $[15,30]$. In this study, in contrast, we evaluated the sensitivity in distinguishing dementia patients from MCI patients and normal subjects. The difference in the targeted patients might have caused the difference in sensitivity scores.

One study has reported the ability of ACE-III to discriminate MCI from normal controls. Matias-Guiu et al. showed that ACE-III scores discriminated between controls and amnestic MCI with high accuracy (AUC, 0.906 by ACE-III memory score) [29]. In our study, the ACE-III-J score (total score) also accurately discriminated MCI from controls (AUC, 0.914). The study of Matias-Guiu et al. detected the difference between amnestic MCI and normal controls. Therefore, the ACE-III memory score was thought to be sensitive enough to discriminate the difference. In this study, the discrimination between MCI (amnestic and non-amnestic) and normal controls was evaluated, and the total score of ACE-III-J was thought to be sensitive enough to differentiate $\mathrm{MCI}$ from normal controls.

Although discrimination of dementia patients from normal controls was reported by several studies $[15,29]$, the discrimination between dementia and MCI patients was evaluated in only one study [29]. Matias-Guiu et al. reported that ACE-III scores discriminated $\mathrm{MCI}$ and dementia patients with high accuracy (AUC, 0.852 by ACE-III total score). In this study, ACE-III-J total score also differentiated dementia patients from those with MCI with high accuracy (AUC, 0.938).

In the cases diagnosed with $\mathrm{MCI}$ in this study, the higher the CDR SoB scores were, the lower the ACE-III scores were. Kim et al. reported that the CDR SoB score 
is useful for predicting the progression to dementia in amnestic MCI individuals. MCI cases with a low ACE-III score may be particularly susceptible to developing dementia in the future [31].

This study has several limitations. First, there were only a few patients with dementia with Lewy bodies, vascular dementia, or frontotemporal dementia in our study. Therefore, we were unable to evaluate the differences in test scores of different dementias. Further study is needed to clarify whether or not it is possible to differentiate dementing diseases by ACE-III-J scores. Second, the participants in this study were outpatients at a university memory center. Third, we diagnosed dementia comprehensively including not only MMSE and HDS-R scores but also total living functions. However, it is undeniable that a potential circularity problem may exist. Thus, the reliability and applicability of ACE-III-J in community samples need further study.

\section{Conclusions}

Regardless of the some limitation, ACE-III-J is an accurate instrument to detect $\mathrm{MCI}$ and dementia. ACE-III-J may be widely useful in clinical practice.

\section{Abbreviations \\ ACE-III: Addenbrooke's Cognitive Examination III; ACE-III-J: The Japanese version of ACE-III; ACE-R: ACE Revised version; ADD: Alzheimer's Disease Dementia; ADL: Activities of Daily Living; AUC: Area Under the Curve; AUROC: Area Under the Receiver Operating Characteristic curve; bvFTD: Behavioral variant Frontotemporal Dementia; CDR: Clinical Dementia Rating; DLB: Dementia with Lewy bodies; HDS-R: Hasegawa Dementia Scale- Revised; ICC: Intraclass Correlation Coefficient; MCl: Mild Cognitive Impairment; MMSE: Mini-mental State Examination; ROC: Receiver Operating Characteristic; VaD: Vascular Dementia}

\section{Acknowledgements}

We would like to thank Professor John Hodges for granting permission to translate the ACE-III into Japanese. Copies of ACE-III (Japanese) can be obtained from the website of "FRONTIER" [32]. We sincerely thank Ms. Yifei Tang and Ms. Sachiko Nagayama for their skillful assistance.

\section{Funding}

This work was supported by research grants from JSPS KAKENHI (Grant Number, 16 K10251 and 17 K10331) and the Zikei Institute of Psychiatry.

\section{Availability of data and materials}

The datasets and analyzed during the current study are available from the corresponding author on reasonable request

\section{Authors' contributions}

ST1 collected the data, analyzed the data, and wrote the paper with ST2. ST2 designed the study, collected the data, and analyzed the data. HY, M Y, MY, $\mathrm{NI}, \mathrm{MH}, \mathrm{TM}$, and OY collected the data. NY supervised the study design, participated in data analysis, and assisted with writing the paper. All authors read and approved the final manuscript.

\section{Ethics approval and consent to participate}

This study adhered to the 1975 Helsinki Declaration of Human Rights. The Internal Ethical Committee of the Okayama University Graduate School of Medicine, Dentistry, and Pharmaceutical Sciences approved this study. We gave all participants written explanations of this research, taking into consideration the cognitive impairment of the participants. After a description of the study, written informed consent was obtained from the subjects who were able to express consent. In addition, written informed consent was obtained from their relatives in all cases.

\section{Consent for publication}

Not applicable.

Competing interests

The authors declare that they have no competing interests.

\section{Publisher's Note}

Springer Nature remains neutral with regard to jurisdictional claims in published maps and institutional affiliations.

Received: 10 September 2018 Accepted: 27 March 2019

Published online: 29 April 2019

\section{References}

1. Tomaszewski Farias S, Cahn-Weiner DA, Harvey DJ, Reed BR, Mungas D, Kramer $\mathrm{JH}$, et al. Longitudinal changes in memory and executive functioning are associated with longitudinal change in instrumental activities of daily living in older adults. Clin Neuropsychol. 2009:23:446-61.

2. Noone P. Addenbrooke's cognitive examination-III. Occup Med. 2015;65: 418-20.

3. Lonie JA, Tierney KM, Ebmeier KP. Screening for mild cognitive impairment: a systematic review. International Journal of Geriatric Psychiatry. 2009;24:902-15.

4. Mathuranath PS, Nestor PJ, Berrios GE, Rakowicz W, Hodges JR. A brief cognitive test battery to differentiate Alzheimer's disease and frontotemporal dementia. Neurology. 2000;55:1613-20.

5. Mioshi E, Dawson K, Mitchell J, Arnold R, Hodges JR. The Addenbrooke's cognitive examination revised (ACE-R): a brief cognitive test battery for dementia screening. International Journal of Geriatric Psychiatry. 2006;21: 1078-85.

6. Hsieh S, Schubert S, Hoon C, Mioshi E, Hodges JR. Validation of the Addenbrooke's cognitive examination III in frontotemporal dementia and Alzheimer's disease. Dement Geriatr Cogn Disord. 2013;36:242-50.

7. Yoshida H, Terada S, Honda H, Kishimoto Y, Takeda N, Oshima E, et al. Validation of the revised Addenbrooke's cognitive examination (ACE-R) for detecting mild cognitive impairment and dementia in a Japanese population. Int Psychogeriatr. 2012;24:28-37.

8. Valcour VG, Masaki KH, Blanchette PL. The phrase: 'no ifs, ands, or buts' and cognitive testing. Lessons from an Asian-American community. Hawaii Med J. 2002;61:72-4

9. Brugnolo A, Nobili F, Barbieri MP, Dessi B, Ferro A, Girtler N, et al. The factorial structure of the mini Mental state examination (MMSE) in Alzheimer's disease. Arch Gerontol Geriatr. 2009;49:180-5.

10. Ganguli M, Ratcliff G, Huff FJ, Belle S, Kancel MJ, Fischer L, et al. Serial sevens versus world backwards: a comparison of the two measures of attention from the MMSE. J Geriatr Psychiatry Neurol. 1990;3:203-7.

11. Newman JC. Copyright and bedside cognitive testing why we need alternatives to the mini-Mental state examination. JAMA Intern Med. 2015 175:1459-60.

12. Machado A, Baeta É, Pimentel P, Peixoto B. Psychometric and normative indicators of the Portuguese version of the Addenbrooke's cognitive examination-III. Preliminary study on a sample of healthy subjects. Acta Neuropsychologica. 2015;13:127-36

13. Matias-Guiu JA, Fernández de Bobadilla R, Escudero G, Pérez-Pérez J, Cortés A, Morenas-Rodríguez $\mathrm{E}$, et al. Validation of the Spanish version of Addenbrooke's cognitive examination III for diagnosing dementia. Neurologia. 2015;30:545-51.

14. Mirza N, Panagioti M, Waheed MW, Waheed W. Reporting of the translation and cultural adaptation procedures of the Addenbrooke's cognitive examination version III (ACE-III) and its predecessors: a systematic review. BMC Med Res Methodol. 2017;17:141

15. Wang BR, Ou Z, Gu XH, Wei CS, Xu J, Shi JQ. Validation of the Chinese version of Addenbrooke's cognitive examination III for diagnosing dementia. International Journal of Geriatric Psychiatry. 2017;32:e173-9.

16. Folstein MF, Folstein SE, McHugh PR. Mini-mental state: a practical method for grading the cognitive state of patients for the clinician. J Psychiatr Res. 1975;12:189-98 
17. Mental SMM. State examination-Japanese. Tokyo: Nihon Bunka Kagakusha; 2012.

18. Katoh S. Development of the revised version of Hasegawa's dementia scale (HDS-R). Japanese Journal of Geriatric Psychiatry. 1991;11:1339-47 (in Japanese).

19. Imai Y, Hasegawa K. The revised Hasegawa's dementia scale (HDS-R) evaluation of its usefulness as a screening test for dementia. Journal of the Hong Kong College of Psychiatrists. 1994;4:20-4.

20. Hughes CP, Berg L, Danziger WL, Coben LA, Martin RL. A new clinical scale for the staging of dementia. Br J Psychiatry. 1982;140:566-72.

21. McKhann GM, Knopman DS, Chertkow H, Hyman BT, Jack CR Jr, Kawas CH, et al. The diagnosis of dementia due to Alzheimer's disease: recommendations from the National Institute on Aging-Alzheimer's Association workgroups on diagnostic guidelines for Alzheimer's disease. Alzheimers Dement. 2011;7:263-9.

22. McKeith IG, Dickson DW, Lowe J, Emre M, O'Brien JT, Feldman H, et al. Diagnosis and management of dementia with Lewy bodies: third report of the DLB consortium. Neurology. 2005;65:1863-72.

23. Rascovsky K, Hodges JR, Knopman D, Mendez MF, Kramer JH, Neuhaus J, et al. Sensitivity of revised diagnostic criteria for the behavioural variant of frontotemporal dementia. Brain. 2011;134:2456-77.

24. Gorelick PB, Scuteri A, Black SE, Decarli C, Greenberg SM, ladecola C, et al. Vascular contributions to cognitive impairment and dementia: a statement for healthcare professionals from the American Heart Association/American Stroke Association. Stroke. 2011;42:2672-713.

25. Albert MS, Dekosky ST, Dickson D, Dubois B, Feldman HH, Fox NC, et al. The diagnosis of mild cognitive impairment due to Alzheimer's disease: recommendations from the National Institute on Aging-Alzheimer's Association workgroups on diagnostic guidelines for Alzheimer's disease. Alzheimers Dement. 2011;7:270-9.

26. American Psychiatric Association. Diagnostic and statistical manual of Mental disorders: DSM-IV-TR. Washington, DC: American Psychiatric Association Publishing; 2000.

27. Cronbach $L$, Meehl PE. Construct validity in psychological tests. Psychol Bull. 1955:52:281-302.

28. Vergara IA, Norambuena T, Ferrada E, Slater AW, Melo F. StAR: a simple tool for the statistical comparison of ROC curves. BMC Bioinformatics. 2008:9:265.

29. Matias-Guiu JA, Cortés-Martínez A, Valles-Salgado M, Rognoni T, FernándezMatarrubia M, Moreno-Ramos T, et al. Addenbrooke's cognitive examination III: diagnostic utility for mild cognitive impairment and dementia and correlation with standardized neuropsychological tests. Int Psychogeriatr. 2017;29:105-13.

30. Elamin M, Holloway G, Bak TH, Pal S. The utility of the Addenbrooke's cognitive examination version three in early-onset dementia. Dement Geriatr Cogn Disord. 2016;41:9-15.

31. Kim JW, Byun MS Sohn BK, Yi D, Seo EH, Choe YM, et al. Clinical dementia rating orientation score as an excellent predictor of the progression to Alzheimer's disease in mild cognitive impairment. Psychiatry Investig. 2017; 14:420-6.

32. Frontier Frontotemporal Dementia Research Group. The University of Sydney, Australia. http://www.ftdrg.org. Accessed 10 Sep 2018.

Ready to submit your research? Choose BMC and benefit from:

- fast, convenient online submission

- thorough peer review by experienced researchers in your field

- rapid publication on acceptance

- support for research data, including large and complex data types

- gold Open Access which fosters wider collaboration and increased citations

- maximum visibility for your research: over $100 \mathrm{M}$ website views per year

At BMC, research is always in progress.

Learn more biomedcentral.com/submissions 